\title{
Gustave Roussy Immune Score Is a Prognostic Factor for Chemotherapy-Naive Pulmonary Adenocarcinoma With Wild-Type Epidermal Growth Factor Receptor
}

\author{
Seigo Minamia, b, Shouichi Ihara ${ }^{\mathrm{a}}$, Kiyoshi Komuta ${ }^{\mathrm{a}}$
}

\begin{abstract}
Background: The Gustave Roussy Immune Score (GRIm-Score) was developed based on the Royal Marsden Hospital (RMH) prognostic score for the purpose of a better patient selection for immunotherapy phase I trials. This scoring system is simply calculated by neutrophil-to-lymphocyte ratio, lactate dehydrogenase (LDH), and serum albumin concentration. The aim of our study was to determine whether GRIm-Score is a practically useful prognostic biomarker for advanced non-small cell lung cancer (NSCLC) patients treated with cytotoxic chemotherapy or epidermal growth factor receptor-tyrosine kinase inhibitor (EGFR-TKI).
\end{abstract}

Methods: This retrospective and single institutional study collected 185 adenocarcinomas without active EGFR mutation, 115 squamous cell carcinomas treated with first-line cytotoxic chemotherapy, and 140 NSCLCs with mutant EGFR treated with first- or second-generation EGFR-TKI monotherapy. These treatments were initiated between July 2007 and March 2018 at our hospital. We compared overall survival (OS) and progression-free survival (PFS) between high and low GRIm-Score groups. Using multivariate Cox proportional hazard analyses, we also found prognostic factors of survival times.

Results: The OS and PFS of low GRIm-Score group were significantly longer than those of high-score group in wild-type EGFR adenocarcinoma (low vs. high; median OS, 18.4 vs. 5.1 months, $\mathrm{P}<0.01$, and median PFS, 5.8 vs. 3.7 months, $\mathrm{P}=0.01)$ and EGFR-mutant NSCLC (median OS, 38.9 vs. 10.4 months, $\mathrm{P}<0.01$, and median PFS, 15.9 vs. 5.0 months, $\mathrm{P}<0.01$ ). Subsequent multivariate analyses detected high GRIm-Score in wild-type EGFR adenocarcinoma as a poor prognostic factor of OS (hazard ratio (HR) 2.20, 95\% CI 1.47 $3.31, \mathrm{P}<0.01$ ), and in the EGFR-mutant NSCLC as a poor prognostic factor of PFS (HR 1.89, 95\% CI 1.00 - 3.55, P = 0.049).

Conclusions: High GRIm-Score was an independent prognostic

Manuscript submitted January 17, 2019, accepted January 25, 2019

a Department of Respiratory Medicine, Osaka Police Hospital, Osaka 5430035, Japan

${ }^{b}$ Corresponding Author: Seigo Minami, Department of Respiratory Medicine, Osaka Police Hospital, 10-31 Kitayama-cho, Tennoji-ku, Osaka-City, Osaka 543-0035, Japan. Email: seigominami@oph.gr.jp

doi: https://doi.org/10.14740/wjon1184 biomarker of OS of first-line cytotoxic chemotherapy for wild-type EGFR adenocarcinoma and of PFS of first- or second-generation EGFR-TKI for EGFR-mutant NSCLC. Therefore, GRIm-Score is not only a specific selection marker for experimental immunotherapy trials, but may also be a promising and useful pretreatment prognostic maker for specific NSCLC subsets in the real-world practice.

Keywords: Gustave Roussy Immune Score; Non-small cell lung cancer; Adenocarcinoma; Squamous cell carcinoma; Neutrophil-tolymphocyte ratio; Serum albumin; Lactate dehydrogenase; Epidermal growth factor receptor

\section{Introduction}

The Gustave Roussy Immune Score (GRIm-Score) was developed with the aim of a better patient selection for phase I trials of immune-checkpoint therapies (ICTs) [1]. This scoring system is based on the Royal Marsden Hospital (RMH) prognostic score, which was also developed for the purpose of improving patient selection for phase I trials of cytotoxic and targeted chemotherapies [2]. These two scoring systems have been validated in the cohorts of patients in phase I trials with various types of solid malignancies [1-3] and non-small cell lung cancer (NSCLC) [4]. Thus, it remains unknown whether these scoring systems are also useful for real-world NSCLC patients. The RMH score is formed by the following three poor prognostic variables: number of metastatic sites $\geq 3$, lactate dehydrogenase $(\mathrm{LDH})>$ upper limit of normal (ULN) range of each institution, and serum albumin concentration $<3.5 \mathrm{~g} / \mathrm{dL}$. In the GRIm scoring system, the number of metastatic sites is replaced with neutrophil-to-lymphocyte ratio (NLR) of $>6$, because the NLR, not the number of metastatic sites, was selected as a significant prognostic factor [1]. The GRIm-Score is simply calculated by a routine blood test. That is the reason why the GRIm-Score is practically more user-friendly than the RMH score.

On the other hand, the Lung Immune Prognostic Index (LIPI) was also recently developed as a specific biomarker for patients with advanced NSCLC treated with ICTs of PD-1/PDL1 inhibitors [5]. This index is based on the combination of derived NLR $(\mathrm{dNLR})>3$ and LDH $>$ ULN. The differences between LIPI and GRIm-Score were only dNLR vs. NLR, the 
cut-off points of dNLR and NLR, and inclusion of serum albumin. Our previous study suggested that the LIPI is a useful prognostic biomarker of cytotoxic chemotherapy for pulmonary adenocarcinoma with wild-type epidermal growth factor receptor (EGFR), and of EGFR-tyrosine kinase inhibitors (TKIs) for NSCLC harboring activated EGFR mutation. As independent prognostic factors of overall survival (OS), our multivariate analyses also detected serum albumin concentration $<3.5 \mathrm{~g} / \mathrm{dL}$ for wild-type EGFR adenocarcinoma treated with chemotherapy and the number of metastatic sites $\geq 2$ for NSCLC with positive EGFR mutation treated with EGFR-TKI (in submission). Considering our previous study, both RMH and GRIm scoring systems are expected to be useful prognostic biomarkers for some subsets of advanced NSCLC.

The aim of this study was to determine whether GRImScore is a practically useful prognostic biomarker for advanced NSCLC patients treated with cytotoxic chemotherapy or EGFR-TKI.

\section{Patients and Methods}

\section{Patients and study design}

This single institutional and retrospective study collected the patients who had received a first- or second- generation EGFRTKI monotherapy (gefitinib, erlotinib or afaitnib) or first-line cytotoxic chemotherapy between July 2007 and March 2018 at our hospital. These patients with pathologically confirmed diagnosis of NSCLC were categorized into the following three groups according to histological and genetic characteristics: 1) wild-type EGFR adenocarcinoma; 2) NSCLC with activated EGFR mutation; and 3) Squamous cell carcinoma. EGFR mutation status was examined by the peptide nucleic acid-locked nucleic acid polymerase chain reaction (PCR) clamp method or cobas EGFR Mutation Test v2 by LSI Medience Cooperation (Tokyo, Japan). Patients with positive ALK rearrangement were excluded. The inclusion criteria were as follows: 1) According to the 7 th edition of Union for International Cancer Control (UICC) tumor-node-metastasis (TNM) classification, C-stage IIIB or IV; 2) For EGFR-positive NSCLC, inoperable and thoracic radiotherapy unfit $\mathrm{C}$-stage IIIA was included; 3) Recurrence after curative-intent surgery or thoracic radiotherapy; 4) Chemotherapy-naive in patients with wild-type EGFR adenocarcinoma and squamous cell carcinoma; and 5) A blood test within 2 weeks before the initiation of the chemotherapy. Patients receiving the first-line pembrolizumab were excluded. The definitions of response to treatment followed Response Evaluation Criteria in Solid Tumors (RECIST) guideline version 1.1. Progression-free survival (PFS) and OS were the times from the initiation of treatments to confirmed progressive disease (PD) or death, and to death, respectively. The data cut-off was the end of November 2018. The GRIm-Score was formed by combination of NLR $(>6=1), \mathrm{LDH}$ ( $>$ ULN range of each center, $225 \mathrm{IU} / \mathrm{L}$ in our hospital $=1)$, and serum albumin $(<3.5 \mathrm{~g} / \mathrm{dL}=1)$. The NLR was calculated by dividing pretreatment neutrophil count by lymphocyte count. Patients were divided into high-score ( 2 or 3 ) and low-score ( 0 or 1$)$ groups [1].

This study protocol and waiver of the written informed consents were approved by the Osaka Police Hospital Ethics Committee, because of retrospective and anonymous data.

\section{Data analysis}

The continuous and categorical variables were described as the median (interquartile range (IQR)) and frequency, and then compared using Mann-Whitney U test and Fisher's exact test, respectively. Survival times were shown as the median $(95 \%$ confidential interval (CI)) and Kaplan-Meier method, and then compared by log-rank test. The predefined independent variables in multivariate Cox proportional hazard analyses followed the previous study of LIPI [5]. These results were expressed by hazard ratio (HR) and 95\% CI. P values of 0.05 or less were considered statistically significant. All statistical analyses were performed with EZR (Saitama Medical Center, Jichi Medical University, Saitama, Japan), which is a graphical user interface for R (R Foundation for Statistical Computing, Vienna, Austria) [6].

\section{Results}

This study collected 185 patients with wild-type EGFR adenocarcinoma, 140 patients with activated EGFR mutation, and 115 patients with squamous cell carcinoma. Thereafter, we sorted these patients into high and low GRIm-Score groups. Patients' background characteristics, treatment details and efficacy, and blood test data were described in Tables 1-3. At the time of data cut-off, the numbers of dead patients and patients who had experienced confirmed PD or death after the first-line chemotherapy were 144 and 166 in wild-type EGFR adenocarcinoma, 71 and 107 in NSCLC harboring positive EGFR mutation, and 82 and 94 in squamous cell carcinoma, respectively.

The OS and PFS of low-score groups were significantly longer than those of high-score groups in wild-type EGFR adenocarcinoma (low vs. high; median OS, 18.4 vs. 5.1 months, $\mathrm{P}<0.01$, and median PFS, 5.8 vs. 3.7 months, $\mathrm{P}=0.01$ ) (Figs. 1a and 2a) and EGFR-mutant NSCLC (median OS, 38.9 vs. 10.4 months, $\mathrm{P}<0.01$, and median PFS, 15.9 vs. 5.0 months, $\mathrm{P}$ $<0.01$ ) (Figs. 1b and 2b). However, in the histological cohort of squamous cell carcinoma, no significant difference was detected in OS (median 12.7 vs. 15.2 months, $\mathrm{P}=0.84$ ) (Fig. 1c) and PFS (median 4.9 vs. 4.5 months, $\mathrm{P}=0.82$ ) (Fig. 2c).

In addition to Eastern Cooperative Oncology Group performance status (ECOG-PS) in all the three histological and genetic cohorts and number of metastatic sites $\geq 2$ in EGFRmutant NSCLC, multivariate Cox proportional hazard analyses detected high GRIm-Score (HR 2.20, 95\% CI 1.47 - 3.31, P $<0.01)$ in wild-type EGFR adenocarcinoma as a poor prognostic factor of OS (Table 4). As for PFS, high GRIm-Score was found in the EGFR-mutant NSCLC cohort as a poor prognostic factor (HR 1.89, 95\% CI $1.00-3.55, \mathrm{P}=0.049$ ), in addition to ECOG-PS $\geq 2$ in wild-type EGFR adenocarcinoma (Table 5). 
Table 1. Baseline Characteristics of Wild-Type EGFR Adenocarcinoma

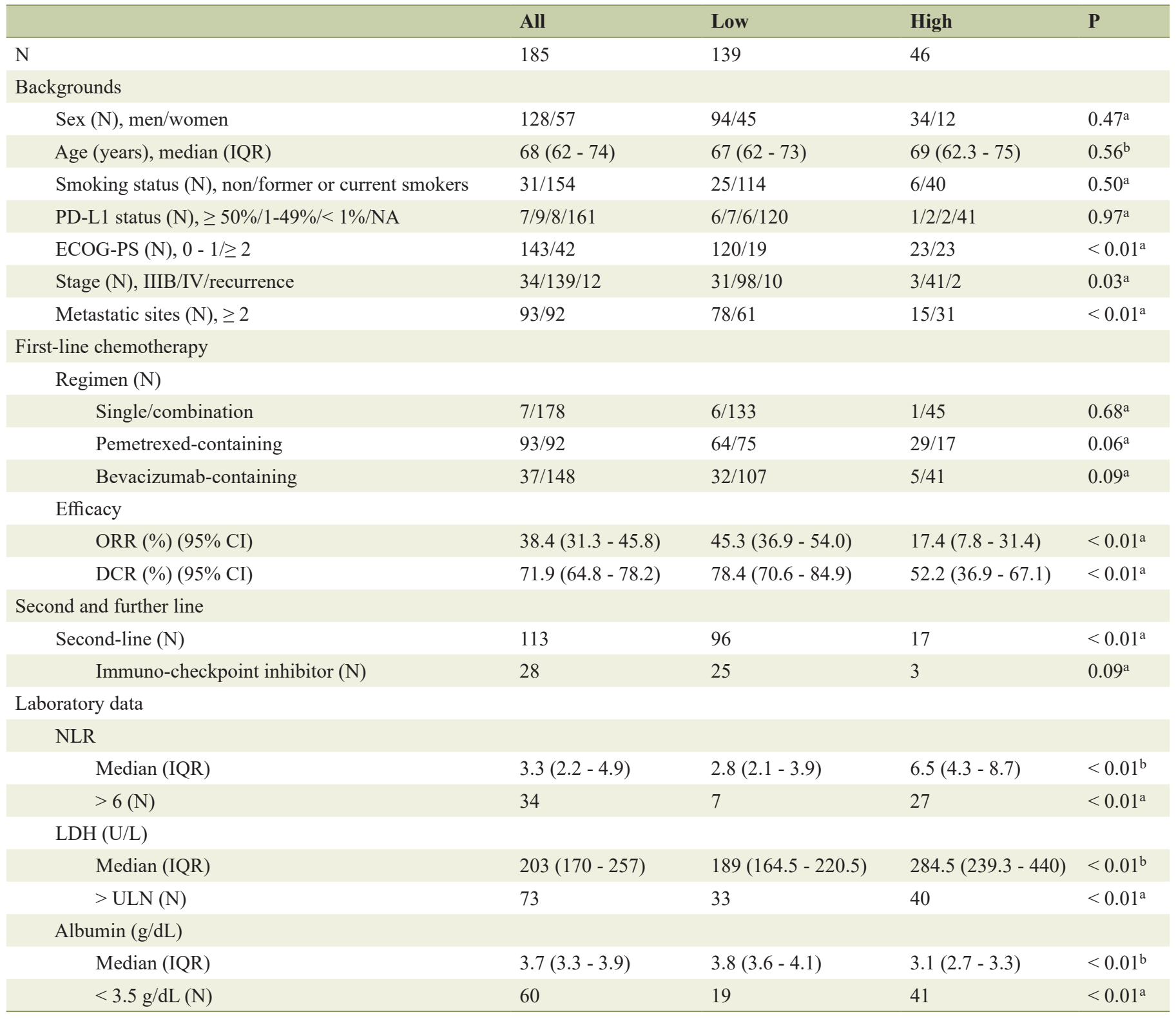

Cl: confidence interval; DCR: disease control rate; ECOG-PS: Eastern Cooperative Oncology Group performance status; IQR: interquartile range; LDH: lactate dehydrogenase; NA: not assessed; NLR: neutrophil-to-lymphocyte ratio; ORR: overall response rate; ULN: upper limit of normal. aFisher's exact test; bMann-Whitney $U$ test.

\section{Discussion}

This study indicates pretreatment GRIm-Score as useful prognostic biomarkers of OS for wild-type EGFR adenocarcinoma and of PFS for EGFR-mutant NSCLC.

The most important finding was that, according to our comparisons of survival times and multivariate analyses, GRIm-Score was an independent prognostic factor of OS of chemotherapynaive wild-type EGFR adenocarcinoma, and of PFS of EGFRTKI monotherapy for EGFR-mutant NSCLC. This biomarker has been validated only in immunotherapy phase I trials [1]. Our study expanded the possibilities of GRIm-Score as a practically available biomarker for NSCLC. Thus, GRIm-Score is not only a specific marker for special cases in experimental trials, but may also be a useful marker for real-world practical settings.

Interestingly, GRIm-Score was not detected as an independent prognostic factor in squamous cell carcinoma. Considering our comparisons of survival curves between high and low GRIm-Score groups, this marker is not expected in this histological subset. Thus, the clinical usefulness of GRIm-Score as a prognostic marker was different among histology, genetic backgrounds, and treatment. A review of our previous studies suggests that, compared with other subsets of NSCLC, squa- 
Table 2. Baseline Characteristics of NSCLC With Active EGFR Mutation

\begin{tabular}{|c|c|c|c|c|}
\hline & All & Low & High & $\mathbf{P}$ \\
\hline $\mathrm{N}$ & 140 & 119 & 21 & \\
\hline \multicolumn{5}{|l|}{ Backgrounds } \\
\hline Age (years), median (IQR) & $73(65-78)$ & $73(65-77)$ & $74(61-78)$ & $0.82^{\mathrm{b}}$ \\
\hline Smoking status $(\mathrm{N})$, non/former or current smokers & $69 / 71$ & $60 / 59$ & $9 / 12$ & $0.64^{\mathrm{a}}$ \\
\hline ECOG-PS $(N), 0-1 / \geq 2$ & $101 / 39$ & $96 / 23$ & $5 / 16$ & $<0.01^{\mathrm{a}}$ \\
\hline Stage $(\mathrm{N}), \mathrm{III} / \mathrm{IV} /$ recurrence & $10 / 96 / 34$ & $10 / 75 / 34$ & $0 / 21 / 0$ & \\
\hline Metastatic sites $(\mathrm{N}), \geq 2$ & 99 & 82 & 17 & $0.31^{\mathrm{a}}$ \\
\hline \multicolumn{5}{|l|}{ EGFR-TKI } \\
\hline \multicolumn{5}{|l|}{ Efficacy } \\
\hline ORR (\%) $(95 \% \mathrm{CI})$ & $64.3(55.8-72.2)$ & $68.9(59.8-77.1)$ & $38.1(18.1-61.6)$ & $0.01^{\mathrm{a}}$ \\
\hline DCR (\%) $(95 \%$ CI $)$ & $82.9(75.6-88.7)$ & $87.4(80.1-92.8)$ & $57.1(34.0-78.2)$ & $<0.01^{\mathrm{a}}$ \\
\hline \multicolumn{5}{|l|}{ Post-EGFR-TKI therapy } \\
\hline Further-line $(\mathrm{N})$ & 69 & 57 & 12 & $0.48^{\mathrm{a}}$ \\
\hline Osimeritinib $(\mathrm{N})$ & 28 & 22 & 6 & $0.37^{\mathrm{a}}$ \\
\hline Immuno-checkpoint inhibitor $(\mathrm{N})$ & 6 & 5 & 1 & $1.00^{\mathrm{a}}$ \\
\hline \multicolumn{5}{|l|}{ Laboratory data } \\
\hline \multicolumn{5}{|l|}{ NLR } \\
\hline Median (IQR) & $2.77(1.93-4.28)$ & $2.52(1.87-3.42)$ & $5.72(3.46-6.93)$ & $<0.01^{\mathrm{b}}$ \\
\hline \multicolumn{5}{|l|}{ Albumin (g/dL) } \\
\hline$<3.5 \mathrm{~g} / \mathrm{dL}(\mathrm{N})$ & 40 & 20 & 20 & $<0.01^{\mathrm{a}}$ \\
\hline
\end{tabular}

Cl: confidence interval; DCR: disease control rate; EGFR: epidermal growth factor receptor; ECOG-PS: Eastern Cooperative Oncology Group performance status; Ex: exon; IQR: interquartile range; LDH: lactate dehydrogenase; NA: not assessed; NLR: neutrophil-to-lymphocyte ratio; ORR: overall response rate; TKI: tyrosine kinase inhibitor; ULN: upper limit of normal. aFisher's exact test; 'Mann-Whitney U test.

mous cell carcinoma has a disadvantage in fewer independent prognostic biomarkers. Except for lymphocyte-to-monocyte ratio [7], none of NLR, modified Glasgow prognostic score [7], LIPI (in submission), or GRIm-Score was selected in patients with squamous cell carcinoma as an independent prognostic marker of OS. Therefore, we should not lump various subsets into the same category as NSCLC, when we develop and validate a biomarker of NSCLC.

Some limitations exist in this study. First, retrospective, single-centered and small sample-sized study might cause a selection bias. Especially, our small sample size might overlook significance of this marker for OS of EGFR-mutant NSCLC. Second, our study did not reflect the new era of immune-on- cology and third-generation EGFR-TKI. Many patients experienced neither ICTs nor osimeritinib. It will be our next challenge to investigate whether this biomarker is useful or not for these new treatments.

\section{Conclusions}

High GRIm-Score was an independent poor prognostic biomarker of OS of first-line cytotoxic chemotherapy for wildtype EGFR adenocarcinoma and of PFS of first- or secondgeneration EGFR-TKI for EGFR-mutant NSCLC. Therefore, GRIm-Score is not only a specific selection marker for experi- 
Table 3. Baseline Characteristics of Squamous Cell Carcinoma



$\mathrm{CI}$ : confidence interval; DCR: disease control rate; ECOG-PS: Eastern Cooperative Oncology Group performance status; IQR: interquartile range; LDH: lactate dehydrogenase; NA: not assessed; NLR: neutrophil-to-lymphocyte ratio; nab-PTX: nanoparticle albumin-bound paclitaxel; ORR: overall response rate; PTX: paclitaxel; ULN: upper limit of normal. 'aFisher's exact test; 'bann-Whitney U test.
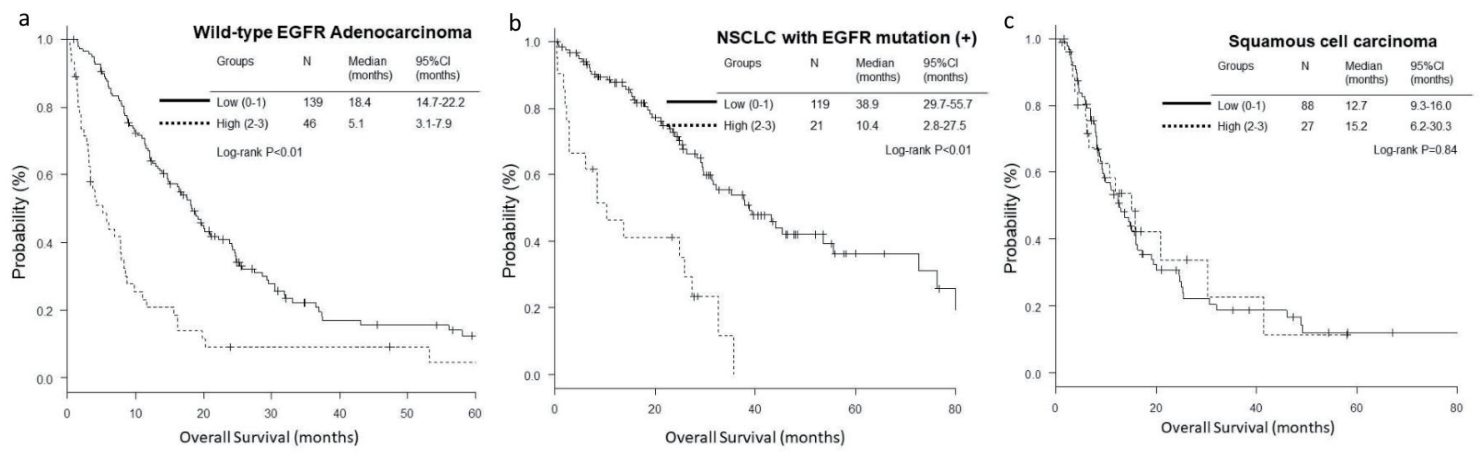

Figure 1. Kaplan-Meier curves of overall survival (OS) according to GRIm-Score. (a) Wild-type EGFR adenocarcinoma. (b) NSCLC with activated EGFR mutation. (c) Squamous cell carcinoma. 

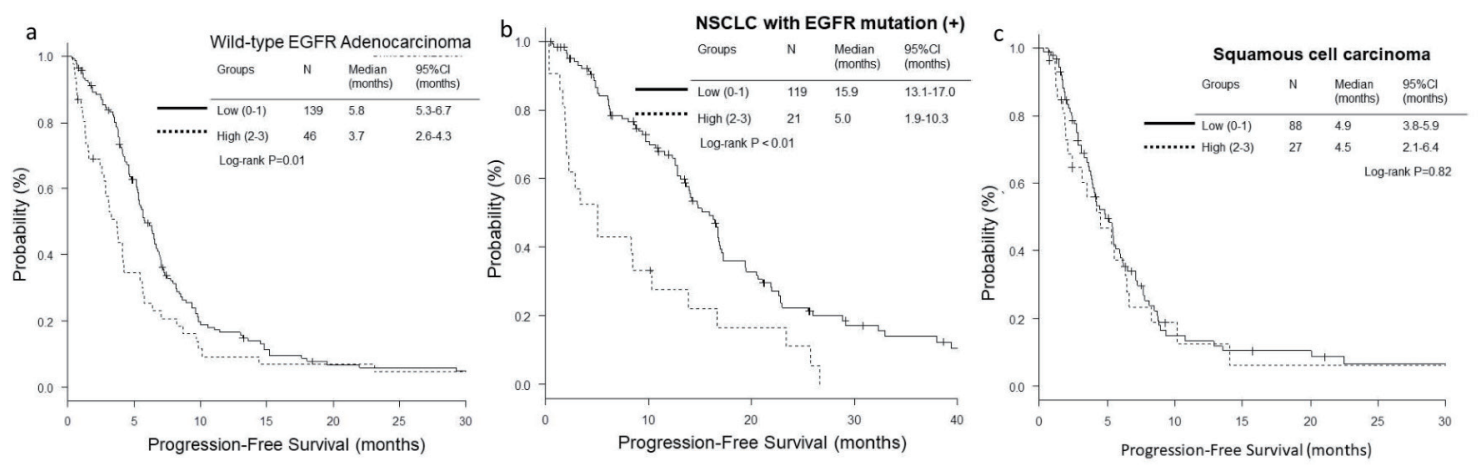

Figure 2. Kaplan-Meier curves of progression-free survival (PFS) according to GRIm-Score. (a) Wild-type EGFR adenocarcinoma. (b) NSCLC with activated EGFR mutation. (c) Squamous cell carcinoma.

mental immunotherapy trials, but may also be a promising and useful pretreatment prognostic maker for specific NSCLC subsets in the real-world practice.

\section{Acknowledgments}

We are grateful to Tsunehiro Tanaka, Kanako Nishimatsu, Shouko Ikuta, Saori Ikebe, Hideyasu Okada and Kazuki Hashimoto at the Department of Respiratory Medicine, Osaka Police Hospital for their medical records, diagnosis, treatment and care of their patients.

\section{Grant Support}

None.

\section{Conflict of Interest}

The authors declare that they have no conflict of interest.

Table 4. Multivariate Analysis of Overall Survival

\begin{tabular}{|c|c|c|c|c|c|c|}
\hline \multirow{2}{*}{ Variable } & Ad, EGFR mt (-) & \multirow{2}{*}{$\mathbf{P}$} & NSCLC, EGFR mt (+) & \multirow{2}{*}{$\mathbf{P}$} & SQ & \multirow{2}{*}{$\mathbf{P}$} \\
\hline & HR $(95 \%$ CI $)$ & & HR $(95 \%$ CI) & & HR $(95 \%$ CI $)$ & \\
\hline \multicolumn{7}{|l|}{ Age, years } \\
\hline$\geq 70$ & $1.31(0.93-1.84)$ & 0.12 & $1.62(0.98-2.69)$ & 0.06 & $0.95(0.59-1.51)$ & 0.81 \\
\hline \multicolumn{7}{|l|}{ Smoking history } \\
\hline \multicolumn{7}{|l|}{ No. of metastatic sites } \\
\hline$<2$ & 1 (Reference) & & 1 (Reference) & & 1 (Reference) & \\
\hline$\geq 2$ & $0.83(0.59-1.18)$ & 0.31 & $2.03(1.13-3.66)$ & 0.02 & $1.51(0.94-2.42)$ & 0.09 \\
\hline \multicolumn{7}{|l|}{ ECOG-PS } \\
\hline First-line & & & 1 (Reference) & & & \\
\hline Second or later line & & & $1.59(0.91-2.78)$ & 0.10 & & \\
\hline \multicolumn{7}{|l|}{ GRIm-Score } \\
\hline Low & 1 (Reference) & & 1 (Reference) & & 1 (Reference) & \\
\hline High & $2.20(1.47-3.31)$ & $<0.01$ & $1.95(0.94-4.04)$ & 0.07 & $0.94(0.54-1.65)$ & 0.84 \\
\hline
\end{tabular}

Ad: adenocarcinoma; Cl: confidence interval; ECOG-PS: Eastern Cooperative Oncology Group performance status; EGFR: epidermal growth factor receptor; HR: hazard ratio; LIPI: lung immune prognostic index; nab-PTX: nanoparticle albumin-bound paclitaxel; NSCLC: non-small cell carcinoma; $\mathrm{SQ}$ : squamous cell carcinoma; TKI: tyrosine kinase inhibitor. 
Table 5. Multivariate Analysis of Progression-Free Survival

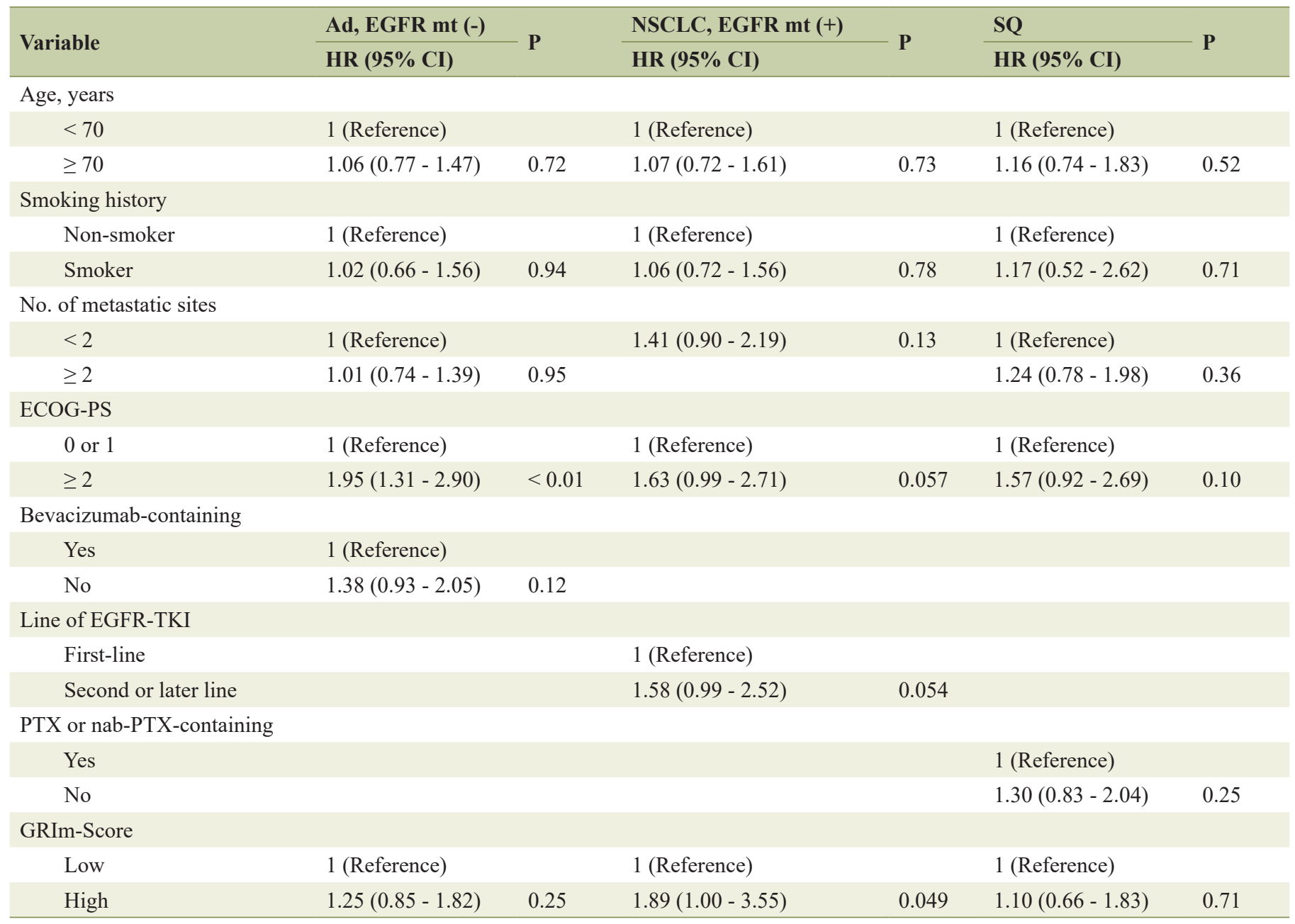

Ad: adenocarcinoma; Cl: confidence interval; ECOG-PS: Eastern Cooperative Oncology Group performance status; EGFR: epidermal growth factor receptor; HR: hazard ratio; LIPI: lung immune prognostic index; nab-PTX: nanoparticle albumin-bound paclitaxel; NSCLC: non-small cell carcinoma; $\mathrm{SQ}$ : squamous cell carcinoma; TKI: tyrosine kinase inhibitor.

\section{References}

1. Bigot F, Castanon E, Baldini C, Hollebecque A, Carmona A, Postel-Vinay S, Angevin E, et al. Prospective validation of a prognostic score for patients in immunotherapy phase I trials: The Gustave Roussy Immune Score (GRIm-Score). Eur J Cancer. 2017;84:212-218.

2. Arkenau HT, Barriuso J, Olmos D, Ang JE, de Bono J, Judson I, Kaye S. Prospective validation of a prognostic score to improve patient selection for oncology phase I trials. J Clin Oncol. 2009;27(16):2692-2696.

3. Garrido-Laguna I, Janku F, Vaklavas C, Falchook GS, Fu S, Hong DS, Naing A, et al. Validation of the Royal Marsden Hospital prognostic score in patients treated in the Phase I Clinical Trials Program at the MD Anderson Cancer Center. Cancer. 2012;118(5):1422-1428.

4. Maymani H, Hess K, Groisberg R, Hong DS, Naing A,
Piha-Paul S, Janku F, et al. Predicting outcomes in patients with advanced non-small cell lung cancer enrolled in early phase immunotherapy trials. Lung Cancer. 2018;120:137-141.

5. Mezquita L, Auclin E, Ferrara R, Charrier M, Remon J, Planchard D, Ponce S, et al. Association of the lung immune prognostic index with immune checkpoint inhibitor outcomes in patients with advanced non-small cell lung cancer. JAMA Oncol. 2018;4(3):351-357.

6. Kanda Y. Investigation of the freely available easy-touse software 'EZR' for medical statistics. Bone Marrow Transplant. 2013;48(3):452-458.

7. Minami S, Ihara S, Komuta K. Pretreatment lymphocyte to monocyte ratio as a prognostic marker for advanced pulmonary squamous cell carcinoma treated with chemotherapy. J Clin Med Res. 2018;10(8):657664. 